$\mathrm{GH}$ abuse in $86 \%$ of men and $60 \%$ of women if a specificity (i.e. probability of false positiveness) of 1:10,000 was used. The authors note, however, that the sensitivity of the test is only sufficient within 2 weeks of $\mathrm{GH}$ administration.

Original article Powrie JK et al. (2007) Detection of growth hormone abuse in sport. Growth Horm IGF Res [doi:10.1016/j.ghir.2007.01.011]

\section{Highly potent PPAR- $\alpha$ agonist versus standard therapy in patients with lipid disorders}

Fibrates are widely prescribed agonists of transcription factor peroxisome proliferatoractivated receptor (PPAR)- $\alpha$, and are used to treat patients with atherogenic dyslipidemia. These drugs cause marked decreases in serum triglyceride levels and moderate increases in HDL cholesterol (HDL-C). Nissen et al. conducted two parallel, multicenter, randomized, double-blind, placebo-controlled trials to determine the safety and efficacy of the novel, highly potent and selective PPAR- $\alpha$ agonist known as LY518674 in patients with lipid disorders.

In the first study, 309 patients with atherogenic dyslipidemia (mean age 54.2 years, $68.9 \%$ men) had baseline HDL-C levels $<45 \mathrm{mg} / \mathrm{dl}$ ( $<50 \mathrm{mg} / \mathrm{dl}$ for women), triglyceride levels $150-600 \mathrm{mg} / \mathrm{dl}$, and LDL cholesterol (LDL-C) levels $\leq 160 \mathrm{mg} / \mathrm{dl}$. After 4 weeks' leadin, participants received LY518674 (10, 25, 50 or $100 \mu \mathrm{g})$, fenofibrate $(200 \mathrm{mg})$ or placebo for 12 weeks. In the second study, LY518674, with or without atorvastatin, was used to treat 276 patients with hypercholesterolemia.

In the atherogenic dyslipidemia study, efficacy of LY518674 in decreasing triglycerides and increasing HDL-C was similar to that of fenofibrate. LY518674 caused a significantly greater increase in LDL-C levels than did fenofibrate. In the hypercholesterolemia study, LY518674 plus atorvastatin increased HDL-C levels and decreased triglyceride levels; however, the reduction in LDL-C levels was comparable to that observed with atorvastatin monotherapy. LY518674 was well tolerated in general, although serum creatinine and alkaline aminotransferase levels increased in some patients.

The authors conclude that LY518674, despite its 10,000 -fold increased potency as a PPAR agonist, is not superior to fenofibrate for control of lipoprotein levels.

Original article Nissen SE et al. (2007) Effects of a potent and selective PPAR- $\alpha$ agonist in patients with atherogenic dyslipidemia or hypercholesterolemia: two randomized controlled trials. JAMA 297: 1362-1373

\section{Use of CXCL10 levels to predict thyroid autoimmunity following IFN- $\alpha$ therapy}

Interferon (IFN)- $\alpha$ therapy is standard for patients with chronic hepatitis $C$ virus infection, although this treatment is associated with the development of thyroid autoimmunity. The chemokine CXC ligand number 10 (CXCL10) is involved in the pathogenesis of several thyroid-related autoimmune diseases. Rotondi and colleagues investigated whether pretreatment levels of CXCL10 predict thyroid autoimmunity development in patients who undergo IFN- $\alpha$ therapy.

This retrospective study included 25 patients (18 males) with hepatitis $\mathrm{C}$ virusrelated chronic hepatitis, and 50 age- and sex-matched healthy controls. The patients were given recombinant IFN- $\alpha-2 a$ three times per week for at least 6 months. Patients were assigned to two groups: those patients who did not develop thyroid autoimmune dysfunction during treatment (Group I, $n=15$ ), and those who did (Group II, $n=10$ ). Levels of CXCL10 were measured before IFN- $\alpha$ therapy, and then at 2, 4 and 6 months after the first treatment session.

Patients in Group I had higher pretreatment serum CXCL10 levels than those in Group II $(308.6 \pm 130.7 \mathrm{pg} / \mathrm{ml}$ versus $191.1 \pm 69.4 \mathrm{pg} / \mathrm{ml}$, $P<0.05)$; this trend was consistent at all time points throughout treatment. A larger proportion of patients in Group II had a favorable response to IFN- $\alpha$ therapy than in Group I ( $90 \%$ versus 33\%).

The authors conclude that pretreatment CXCL10 measurement can identify those patients more likely to respond well to IFN- $\alpha$ therapy and to develop thyroid autoimmunity; these patients should be carefully monitored with thyroid surveillance throughout treatment.

\footnotetext{
Original article Rotondi M et al. (2007) Serum CXCL10 levels and occurrence of thyroid dysfunction in patients treated with interferon- $\alpha$ therapy for hepatitis $C$ virusrelated hepatitis. Eur J Endocrinol 156: 409-414
} 